\author{
Rainer Surges \\ Martin Schubert \\ Ansgar Berlis \\ Cornelius Weiller \\ Andreas Hetzel \\ Wolf-Dirk Niesen
}

\section{Spinal subarachnoid hemorrhage due to ruptured solitary aneurysm at thoracolum- bar level with fatal outcome}

Sirs: Spinal subarachnoid hemorrhage (SAH) acounts for less than $1 \%$ of SAHs. Cerebral complications are uncommon and moderate in severity. We report on a 30 year old woman suffering from severe cerebral vasospasms, without subarachnoid blood in successive cranial CT scans, due to spinal SAH caused by a ruptured solitary spinal aneurysm of unknown etiology.

The patient suffered from a graft-versus-host reaction of her transplanted kidney. Under

R. Surges - M. Schubert $\cdot$ C. Weiller

A. Hetzel · W.-D. Niesen $(\square)$

Dept. of Neurology

University Clinics of Freiburg

Breisacherstr. 64

79106 Freiburg, Germany

Tel.: +49-761/270-5157

Fax: +49-761/270-5328

E-Mail: wolf-dirk.niesen@uniklinikfreiburg.de

A. Berlis

Dept. of Neuroradiology

University Clinics of Freiburg

Breisacherstr. 64

79106 Freiburg, Germany immunosuppression, she developed recurrent pneumonias and a septicemia with unknown germ that were treated by intravenous antibiotics. In the further course, she suddenly presented with headache, back pain and progressive behavioural disturbances. Cranial CT at this time was normal. Three lumbar punctures revealed a hemorrhagic CSF that was considered to be artificially bloody. Because of increasing paraparesis and rectal incontinence, the patient was referred to our clinic one week later. On admission, she was in poor clinical condition with reduced consciousness, no speech production, nuchal rigidity and paraplegia lasting for more than 24 hours. Cranial CT was normal, whereas cranial MRI showed bilateral MCA-infarction on diffusionweighted MRI (Fig. 1A). Spinal MRI revealed a ventrally located intraspinal circular tumour at the level of the twelfth thoracic vertebra with an intradural T1-hyperintensive signal in the thoracolumbar canal (Fig. 1B). Suboccipital puncture yielded a hemorrhagic CSF (2540 cells per $\mu \mathrm{l})$. Conventional panangiography revealed a solitary aneurysm $(8 \times 15 \mathrm{~mm})$ originating from the descending branch of the anterior spinal artery at the level of the twelfth thoracic vertebra (Fig. 1C) as well as generalised, severe cerebral vasospasms. Despite immediate triple-H- and nimodipine-therapy, CT 12h later demonstrated large bilateral MCAinfarction, but was still without any cerebral SAH (Fig. 1D). Because of the infavorable prognosis, palliative treatment was carried out until death due to a pneumogenic sepsis 23 days later.

To date, 20 cases of spinal SAH due to a rupture of isolated spinal aneurysms have been reported
[1-2, 4-16]. Of the eight reported patients with thoracolumbar aneurysms, all had an acute onset of pain in the thoracolumbar region $[1,8,11,13,15]$. Three also had paraparesis $[1,8]$. Six of the eight patients were treated surgically $[8,11,13,15]$ whilst the other two had a conservative treatment [1]. All patients recovered either without or with minor neurological deficits. The authors found two dissecting aneurysms [8], an inflammation within the vessel wall in two cases $[1,11]$ and no evident etiology in the other four reports $[1,8,13,15]$. In our patient, general autopsy did not reveal any underlying systemic (e.g. polyarteriitis nodosa, connective tissue disorder, syphilis), cerebral or further spinal vascular pathology. Since the SAH was related in timing to systemic infection in the context of immunosuppression, the aneurysm may result from a local inflammation. Morphologically, inflammatory aneurysms are characterised by proliferation of the tunica intima and media as well as inflammatory infiltrates. Infectious agents may be detected within the vessel wall $[4,11]$. However, the aneurysm in our patient only yielded a prominent fibroblastic proliferation without inflammatory infiltrates (Fig. 2). Additional analysis of blood and CSF samples did not contain any germs and all serological tests were negative. Thus the inflammatory etiology remains speculative. In summary, we present the case of a young woman who suffered from symptomatic cerebral vasospasms due to spinal SAH caused by a ruptured solitary spinal aneurysm. Pathogenesis of cerebral vasospasm is unclear, but is most probably due to a distant inflammatory response caused by the subarachnoid blood [3]. Although there have been a few 
Fig. 1 (A) Diffusion-weighted cranial MRI showing subacute infarctions within the region of the right MCA at admission. (B) Spinal MRI revealed a ventral intraspinal tumor at the level of the twelfth thoracic vertebra. (C) Conventional angiography showed a solitary aneurysm originating from the descending branch of the anterior spinal artery at the level of the twelfth thoracic vertebra. (D) Bilateral infarctions in both MCA-territories on CT 12 hours after admission. Note the thickened skull bone related to renal osteopathy
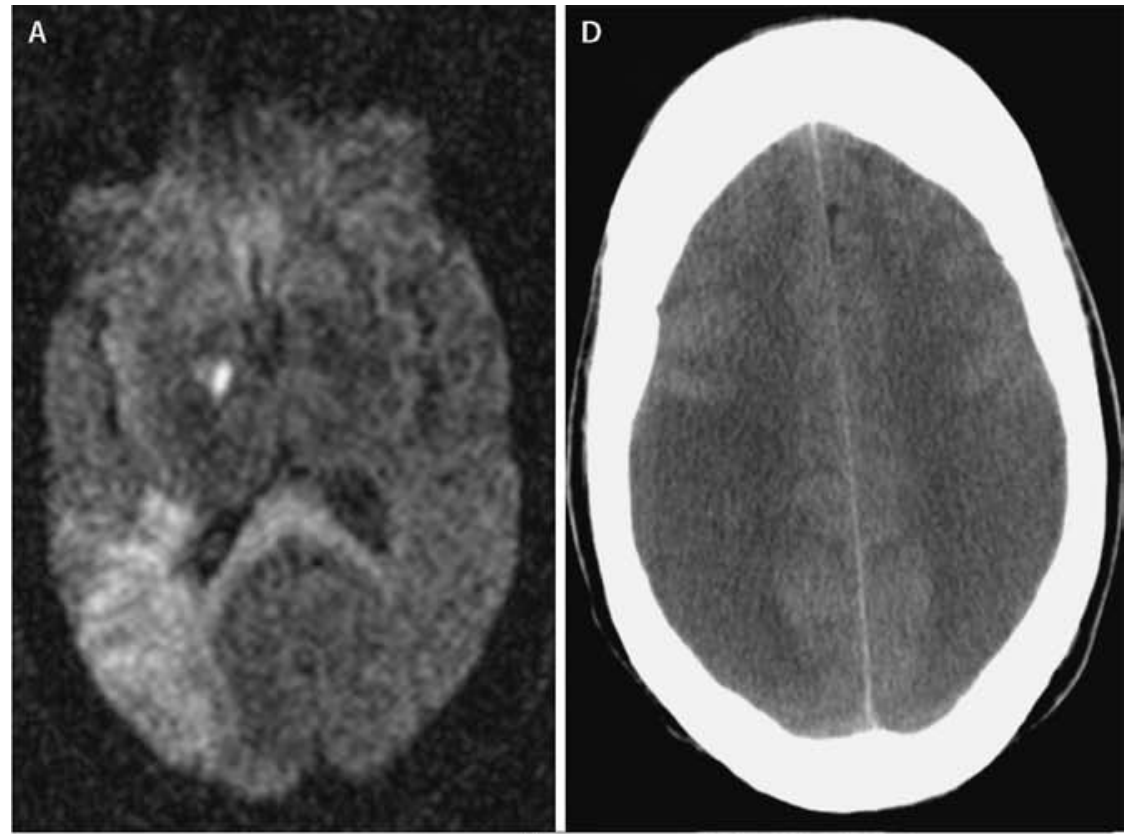

B

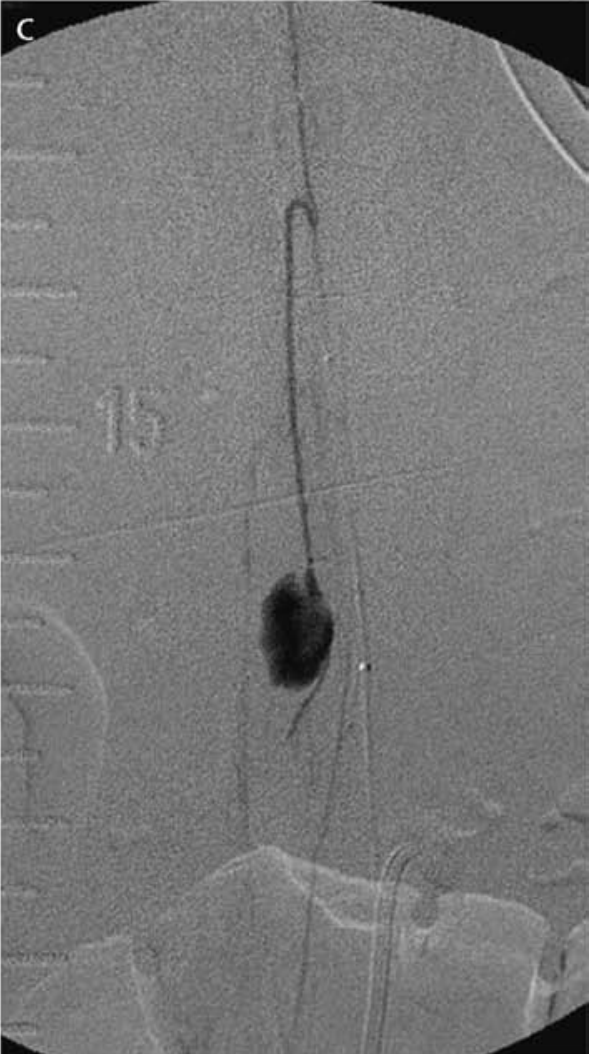

c other cases $[10,14]$, this is the first report with generalised severe cerebral vasospasms leading to extensive cerebral infarction due to a spinal SAH without detectable subarachnoid blood on cranial CT. 

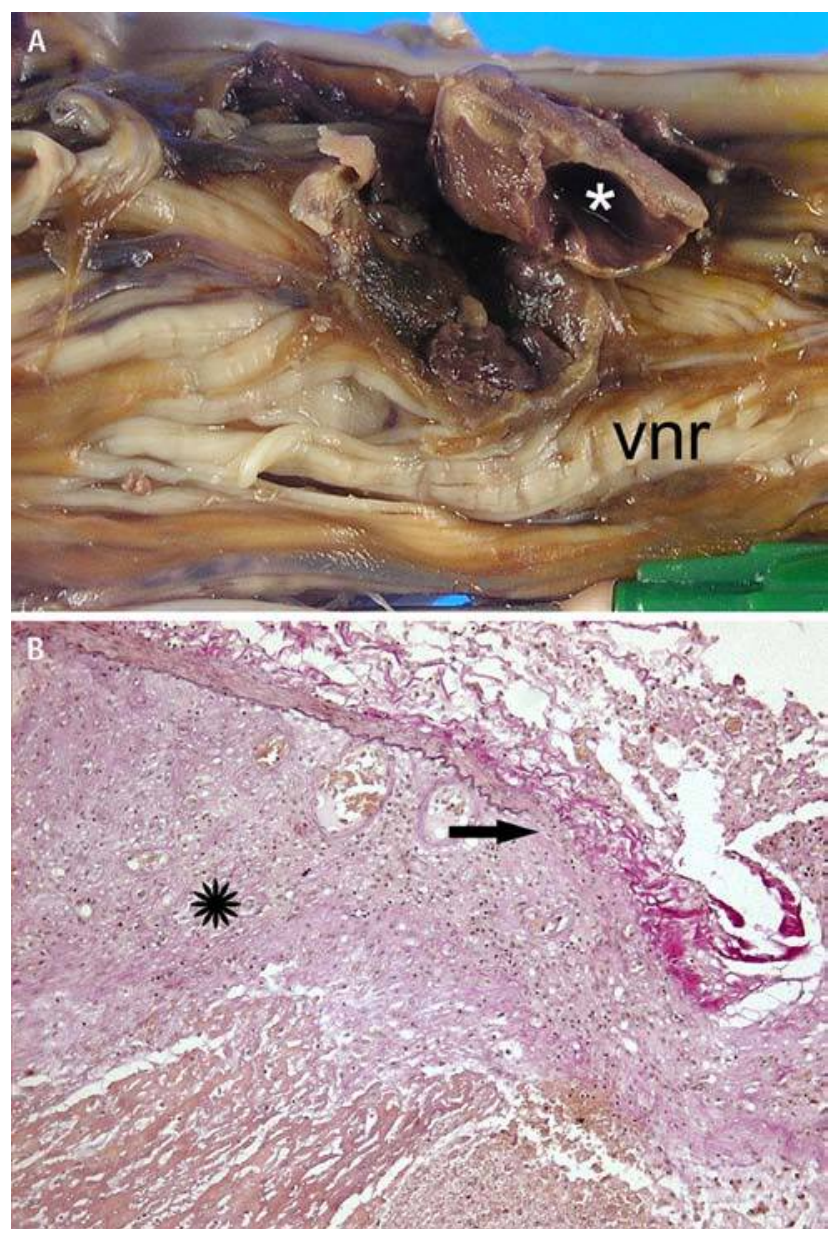

Fig. 2 (A) Macroscopic view on the aneurysm at the ventral part of the spinal cord. Note the cavity of the aneurysma (asterisk). Vnr: ventral nerve roots. (B) Part of the wall of the saccular aneurysm showing brisk interruption of the elastic lamina (arrow), increased kollagen fibers (red) and a broad fibrous connection to the surface of spinal cord (asterisk). Elastica van Gieson staining, magnification $\times 100$

Acknowledgement We thank Dr. Klaus Müller (Department of Neuropathology) for critically reading the manuscript and providing us the macroscopic/microscopic pictures.

\section{References}

1. Berlis A, Scheufler KM, Schmahl C, Rauer S, Gotz F, Schumacher M (2005) Solitary spinal artery aneurysms as a rare source of spinal subarachnoid hemorrhage: potential etiology and treatment strategy. AJNR Am J Neuroradiol 26:405-410
5. Goto Y, Kamijyo Y, Yonekawa Y, Kikuchi H (1988) Ruptured aneurysm of the posterior spinal artery of the upper cervical spinal cord: case report. Neurosurgery 22:558-560

6. Henson RA, Croft PB (1956) Spontaneous spinal subarachnoid haemorrhage. Q J Med 25:53-66

7. Kawamura S, Yoshida T, Nonoyama Y, Yamada M, Suzuki A, Yasui N (1999) Ruptured anterior spinal artery aneurysm: a case report. Surg Neurol 51:608-612

8. Massand MG, Wallace RC, Gonzalez LF, Zabramski JM, Spetzler RF (2005) Subarachnoid hemorrhage due to isolated spinal artery aneurysm in four patients. AJNR Am J Neuroradiol 26:2415-2419

9. Mohsenipour I, Ortler M, Twerdy K, Schmutzhard E, Attlmayr G, Aichner F (1994) Isolated aneurysm of a spinal radicular artery presenting as spinal subarachnoid haemorrhage. J Neurol Neurosurg Psychiatry 57:767-768

10. Moore DW, Hunt WE, Zimmerman JE (1982) Ruptured anterior spinal artery aneurysm: repair via a posterior approach. Neurosurgery 10:626-630

11. Rengachary SS, Duke DA, Tsai FY, Kragel PJ (1993) Spinal arterial aneurysm: case report. Neurosurgery 33:125-129

12. Saunders FW, Birchard D, Willmer J (1987) Spinal artery aneurysm. Surg Neurol 27:269-272

13. Smith BS, Penka CF, Erickson LS, Matsuo F (1986) Subarachnoid hemorrhage due to anterior spinal artery aneurysm. Neurosurgery 18:217-219

14. Vincent FM (1981) Anterior spinal artery aneurysm presenting as a subarachnoid hemorrhage. Stroke 12:230232

15. Vishteh AG, Brown AP, Spetzler RF (1997) Aneurysm of the intradural artery of Adamkiewicz treated with muslin wrapping: technical case report. Neurosurgery 40:207-209

16. Yonas H, Patre S, White RJ (1980) Anterior spinal artery aneurysm. J Neurosurg 53:570-573 